\title{
The Effect of Exit Strategy on Optimal Portfolio Selection with Birandom Returns
}

\author{
Guohua Cao and Dan Shan \\ School of Economics and Business Administration, Chongqing University, Room 240, No. 12, Dormitory Building, Area A, \\ Shapingba District, Chongqing 400030, China \\ Correspondence should be addressed to Dan Shan; 332167699@qq.com
}

Received 18 October 2012; Revised 15 April 2013; Accepted 17 April 2013

Academic Editor: Xiaojun Wang

Copyright (C) 2013 G. Cao and D. Shan. This is an open access article distributed under the Creative Commons Attribution License, which permits unrestricted use, distribution, and reproduction in any medium, provided the original work is properly cited.

\begin{abstract}
The aims of this paper are to use a birandom variable to denote the stock return selected by some recurring technical patterns and to study the effect of exit strategy on optimal portfolio selection with birandom returns. Firstly, we propose a new method to estimate the stock return and use birandom distribution to denote the final stock return which can reflect the features of technical patterns and investors' heterogeneity simultaneously; secondly, we build a birandom safety-first model and design a hybrid intelligent algorithm to help investors make decisions; finally, we innovatively study the effect of exit strategy on the given birandom safetyfirst model. The results indicate that (1) the exit strategy affects the proportion of portfolio, (2) the performance of taking the exit strategy is better than when the exit strategy is not taken, if the stop-loss point and the stop-profit point are appropriately set, and (3) the investor using the exit strategy become conservative.
\end{abstract}

\section{Introduction}

During decades of development of Chinese stock market, plentiful practice has proved that some technical patterns occur repeatedly in stock market. Stocks with some technical patterns are much more likely to profit than those with other technical patterns. Douglas [1] pointed out that investors should distinguish the recurring technical patterns, summarize the laws, and use them to choose stocks, which can greatly improve the performance of profit. If investors can consider the features of technical patterns and investors' heterogeneity in estimating the stock return, then they can not only enhance the accuracy of estimation but also make it conform to the actual decision-making process of investors more. So a natural question is how to reflect the features of these technical patterns and investors' heterogeneity in the stock return? To the best of our knowledge, there has been no literature in this respect. In this paper, we try to answer this question for the first time.

Estimating the stock return has always been one of the theoretical hot issues, and much progress has been made in this area. The stock return was assumed to follow the normal distribution [2], which had been verified not to coincide with the facts by a great deal of afterward researches. In order to describe the stock return better, the Logistic distribution [3], Student- $t$ distribution [4], and stable Paretian distribution [5] were put forward. Later, fuzzy random distribution [6] and random fuzzy distribution [7] were used separately via combining randomness with fuzziness. In $[8,9]$, the stock return was assumed to follow birandom distribution. All of the above distributions have certain theoretical meaning, but there are two limitations: one is that they do not consider the stock's technical patterns, thus hiding the particularity of technical patterns, and the other is that investors' heterogeneity cannot be reflected from the above distributions. In this paper, we propose a method to estimate the stock return and the stock return gained finally can be indicated with a birandom variable and thus solving the above two limitations well.

After the stock return being determined, it is important to choose a portfolio model to help investors make decisions. Roy [10] proposed the safety-first model. The main 
point was investors would try to make the probability of a disaster smallest when allocating asset. Pyle and Turnovsky [11] summarized the three forms of the safety-first rule. A safety-first chance constrained model [12] was proposed by combining the safety-first rule with chance constraint. Based on the safety-first rule, a birandom chance constrained model [8] and a birandom expected value model [9] were put forward when the stock return follows birandom distribution. However, the model in [8] just takes the budget constraint into consideration without considering the effect of a disaster. The model in [9] uses the expected value to describe the profit, but the expected value model is only applied to risk neutral investors not to risk aversion and risk loving ones. In this paper, a birandom safety-first model is acquired by combining the two models in $[8,9]$. In fact, the new model not only considers the effect of disaster, but also can apply to all investors through parameters adjustment.

As we all know, there are two important parts in investment process: how to buy and how to sell. The theory of portfolio selection is widely used to help us decide the proportion of portfolio, which to solve the problem of how to buy. However, to the best of our knowledge, there is no research on the effect of selling strategy on portfolio selection. Exit strategy proposed in behavioral finance has been proved to be an effective selling strategy, and we try to study the effect of exit strategy on birandom portfolio selection in this paper.

The rest of the paper is organized as follows: in Section 2, we illustrate how to describe the stock return using a birandom variable. In Section 3, we propose a birandom safetyfirst model, a hybrid intelligent algorithm, and a numerical example. In Section 4, we study the effect of exit strategy on birandom portfolio selection. Finally, in Section 5, we draw the conclusion.

\section{Estimate the Stock Return}

In the following paragraphs, firstly we will introduce the birandom theory, secondly show how to describe the stock return using a birandom variable, and finally give a numerical example.

\subsection{Birandom Theory}

Definition 1 (Jin and Bao-Ding [13]). A birandom variable $\varepsilon$ is a mapping from a probability space $\left(\Omega, A, P_{r}\right)$ to a collective of random variables such that for any Borel subsets $B$ of the real line $R$, the induced function $P_{r}(\varepsilon(\omega) \in B$ ) is a measure function with respect to $\omega$.

Example 2. Let $\Omega=\left(\omega_{1}, \omega_{2}\right)$, and $P_{r}\left(\omega_{1}\right)=a, P_{r}\left(\omega_{2}\right)=b$. Assume that $\varepsilon$ is a function on $\left(\Omega, A, P_{r}\right)$ as follows:

$$
\varepsilon(\omega)= \begin{cases}\varepsilon_{1}, & \text { if } \omega=\omega_{1}, \\ \varepsilon_{2}, & \text { if } \omega=\omega_{2},\end{cases}
$$

where $\varepsilon_{1}$ and $\varepsilon_{2}$ are random variables. Then $\varepsilon$ is a birandom variable according to Definition 1. In Example 2, $\Omega$ represents stock, $\omega_{1}$ represents stock rising, $\omega_{2}$ represents stock falling, $a$ and $b$ represent the probabilities of stock rising and falling, respectively, and $\varepsilon_{1}$ represents profit distribution, $\varepsilon_{2}$ represents loss distribution.

Definition 3 (Jin and Bao-Ding [13]). Let $\varepsilon=\left(\varepsilon_{1}, \varepsilon_{2}, \ldots, \varepsilon_{n}\right)$ be a birandom vector on $\left(\Omega, A, P_{r}\right)$ and $f: R^{n} \rightarrow R^{m}$ be a vector-valued Borel measurable function. Then the primitive chance of birandom event characterized by $f(\varepsilon) \leq 0$ is a function from $(0,1]$ to $[0,1]$, defined as

$$
\begin{aligned}
& \operatorname{Ch}\{f(\varepsilon) \leq 0\}(\alpha) \\
& \quad=\sup \left\{\beta \mid P_{r}\left\{\omega \in \Omega \mid P_{r}\{f(\varepsilon(\omega)) \leq 0\} \geq \beta\right\} \geq \alpha\right\} .
\end{aligned}
$$

2.2. Method to Estimate the Stock Return. Assuming that an investor selects stock $S$ in accordance with technical pattern $T$, a new method is proposed to estimate the return distribution of stock $S$. The specific steps are listed as follows.

Step 1. Calculate the probabilities of stock rising and falling after the appearance of technical pattern $T$. The method is to select $n$ representative stocks and inspect the occurrence number of technical pattern $T$ of the $n$ stocks during a certain period, and then figure out the numbers of price rising and falling separately. When the sample size is large enough, it is reasonable to substitute the frequencies of stock rising and falling for the probabilities of stock rising and falling. Through the comparison of probabilities, the technical patterns can be divided into three types where the probability of rising is larger than, equal to, and smaller than the probability of falling. What we care for is the first type, and it is named as the advanced technical pattern.

Step 2. Because stock $S$ has the features of technical pattern $T$, we substitute its probabilities of rising and falling by the corresponding probabilities in Step 1. Thus $P_{r}\left(\omega_{1}\right)=a$, $P_{r}\left(\omega_{2}\right)=b$ in Example 2 are obtained.

Step 3. When stock $S$ rises, we estimate the profit distribution of stock $S$. When stock $S$ falls, we estimate the loss distribution of stock $S$. Thus $\varepsilon_{1}$ and $\varepsilon_{2}$ in Example 2 are obtained. Here the following two things should be emphasize.

(1) $\varepsilon_{1}$ and $\varepsilon_{2}$ can be obtained from the investors' experience, the stock historical data, and the existing stock forecast methods. In this paper, we assume that $\varepsilon_{1}$ and $\varepsilon_{2}$ are random variables.

(2) Different investors have different opinions and judgments on the stock market; therefore, different assumptions of $\varepsilon_{1}$ and $\varepsilon_{2}$ can demonstrate the heterogeneity of investors.

From the above, the stock return is quantified by the birandom variable in Example 2. Among them, the formulas $P_{r}\left(\omega_{1}\right)=a, P_{r}\left(\omega_{2}\right)=b$ demonstrate the features of technical patterns and the distributions of $\varepsilon_{1}$ and $\varepsilon_{2}$ reflect the investors' heterogeneity.

2.3. A Numerical Example. Because stock $S$ follows the birandom distribution in Example 2, we can calculate $a, b, \varepsilon_{1}$, and $\varepsilon_{2}$ in the following steps. 
Step 1 (calculate $a$ and $b$ ). Suppose that we select fifty representative stocks and the appearance numbers of technical pattern $T$ of the fifty stocks are equal to one hundred during the last year. If price goes up 60 times and down 40 times, then the probabilities of stock $S$ rising and falling are equal to 0.6 and 0.4 , respectively, so $a=0.6$ and $b=0.4$.

Step 2 (the determination of $\varepsilon_{1}$ and $\varepsilon_{2}$ ). After selecting stock $S$ based on technical pattern $T$, the investor concludes, by various analytical methods such as fundamental analysis and technical analysis, that the profit of stock $S$ should be distributed uniformly in the interval $[0,7 \%]$. Therefore, the profit distribution $\varepsilon_{1}$ can be assumed to follow the uniform distribution $U[0,7 \%]$. Similarly, we can also assume that the loss distribution $\varepsilon_{2}$ follows the uniform distribution $U[-9 \%, 0]$.

Remark 4. The detailed statistic process in Step 1 is not included in the paper.

Remark 5. $\varepsilon_{1}$ and $\varepsilon_{2}$ can be arbitrary random variable and different distributions of $\varepsilon_{1}$ and $\varepsilon_{2}$ have no effect on the validity of the model and the algorithm given in the following paragraphs. Here, the assumption of uniform distribution is to simplify calculation.

Now we get the distribution of stock $S$. The distribution is given by the following.

Example 6. $\Omega=\left(\omega_{1}, \omega_{2}\right)\left(\Omega\right.$ represents stock $S, \omega_{1}$ represents stock $S$ rising, and $\omega_{2}$ represents stock $S$ falling), and $P_{r}\left(\omega_{1}\right)=$ $0.6, P_{r}\left(\omega_{2}\right)=0.4$,

$$
\varepsilon(\omega)= \begin{cases}\varepsilon_{1}, & \text { if } \omega=\omega_{1}, \\ \varepsilon_{2}, & \text { if } \omega=\omega_{2},\end{cases}
$$

where $\varepsilon_{1}$ follows $U[0,7 \%]$ and $\varepsilon_{2}$ follows $U[-9 \%, 0]$.

\section{Birandom Safety-First Model}

\subsection{Assumptions of the Birandom Safety-First Model}

(A1) The investor selects $n$ stocks based on $n$ kinds of technical patterns. Let $m_{i}$ and $x_{i}$ denote the $i$ th stock and the investment proportion of the $i$ th stock, $i=$ $1,2, \ldots, n$, respectively. And the return of stock $m_{i}$ follows the birandom distribution in Example 2.

(A2) Stocks can be infinite subdivision. the market does not allow shorting and there is no limitation on the transaction cost.

3.2. Measures of the Profit and Risk. In this paper, the chance that return of total asset (RTA) is no less than some value is used to measure the profit which is given by

$$
\text { Ch }\left\{x_{1} m_{1}+x_{2} m_{2}+\cdots+x_{n} m_{n} \geq m\right\}\left(\alpha_{1}\right) .
$$

The chance that RTA is no less than a disaster level is regarded to measure the risk which is given by

$$
\operatorname{Ch}\left\{x_{1} m_{1}+x_{2} m_{2}+\cdots+x_{n} m_{n} \geq n\right\}\left(\alpha_{2}\right) \geq \beta .
$$

3.3. Birandom Safety-First Model. Using the measures of the profit and risk given above, the birandom safety-first model is given by

$$
\begin{array}{ll}
\max & \operatorname{Ch}\left\{x_{1} m_{1}+x_{2} m_{2}+\cdots+x_{n} m_{n} \geq m\right\}\left(\alpha_{1}\right), \\
\text { s.t. } & \operatorname{Ch}\left\{x_{1} m_{1}+x_{2} m_{2}+\cdots+x_{n} m_{n} \geq n\right\}\left(\alpha_{2}\right) \geq \beta, \\
& \sum_{i=1}^{n} x_{i}=1, \\
& x_{i} \geq 0, \quad i=1,2, \ldots, n,
\end{array}
$$

where $m$ represents the profit, $n$ represents the disaster level, $\alpha_{1}$ and $\alpha_{2}$ represent the investor's tolerance, and $\beta$ is the lower bound of the chance that RTA is not less than some disaster level when the tolerance is $\alpha_{2}$.

The economic meanings of model (6) are listed as follows.

(1) The constraint condition

$$
\sum_{i=1}^{n} x_{i}=1
$$

means that the capital cannot exceed the budget.

(2) The constraint condition

$$
x_{i} \geq 0, \quad i=1,2, \ldots, n
$$

means that the market does not allow shorting.

Remark 7. Model (6) can be used for all investors as long as choosing the right parameters $m, n, \alpha_{1}, \alpha_{2}, \beta$.

Remark 8. When the values of $n$ and $\alpha_{2}$ are fixed, the value of $\beta$ is in inverse ratio with the risk tolerance ability. (Proof is described in the appendix.)

Remark 9. In the case that the return distribution of stock $m_{i}$ is a random variable, model (6) is reduced to the special one:

$$
\begin{array}{ll}
\max & P_{r}\left\{x_{1} m_{1}+x_{2} m_{2}+\cdots+x_{n} m_{n} \geq m\right\}, \\
\text { s.t. } & P_{r}\left\{x_{1} m_{1}+x_{2} m_{2}+\cdots+x_{n} m_{n} \geq n\right\} \geq \beta, \\
& \sum_{i=1}^{n} x_{i}=1, \\
& x_{i} \geq 0, \quad i=1,2, \ldots, n .
\end{array}
$$

3.4. Hybrid Intelligent Algorithm. Because of the uncertainty of birandom variable, it is hard to analytically solve model (6). To provide a general solution to model (6), we design a hybrid intelligent algorithm integrating genetic algorithm (GA) and birandom simulation.

3.4.1. Birandom Simulation. The technique of birandom simulation is applied to compute (4) and (5). The specific steps are listed as follows. 
Algorithm 10 (birandom simulation for (4)).

Step 1. Generate $w_{1}, w_{2}, \ldots, w_{N}$ from $\Omega$ according to the probability measure $P_{r}$.

Step 2. Compute the probability $\beta_{n}=P_{r}\left\{x_{1} m_{1}+x_{2} m_{2}+\cdots+\right.$ $\left.x_{n} m_{n}\right\} \geq m$ for $n=1,2, \ldots, N$, respectively, by stochastic simulation.

Step 3. Set $N^{\prime}$ as the integer part of $\alpha_{1} N$.

Step 4. Return the $N^{\prime}$ th largest element $\beta^{*}$ in $\left(\beta_{1}, \beta_{2}, \ldots, \beta_{N}\right)$.

Step 5. $\beta^{*}$ is the value of (4).

Algorithm 11 (birandom simulation for (5)).

Step 1. Set $l=1$.

Step 2. Generate $w_{1}, w_{2}, \ldots, w_{M}$ from $\Omega$ according to the probability measure $P_{r}$.

Step 3. Compute the probability $\beta_{n}=P_{r}\left\{x_{1} m_{1}+x_{2} m_{2}+\cdots+\right.$ $\left.x_{n} m_{n}\right\} \geq n$ for $n=1,2, \ldots M$, respectively, by stochastic simulation.

Step 4. Set $M^{\prime}$ as the integer part of $\alpha_{2} M$.

Step 5. Return the $M^{\prime}$ th largest element $\beta^{*}$ in $\left(\beta_{1}, \beta_{2}, \ldots, \beta_{M}\right)$.

Step 6. If $\beta^{*}$ is larger than $\beta$, then $l=l * 1$, else $l=l * 0$.

Step 7. If $l=1$, then return Yes, else return No. Here, Yes means the investment proportion $x$ satisfies (5), and No means that $x$ does not satisfy (5).

3.4.2. Genetic Algorithm. The specific steps of genetic algorithm are described in [8].

\subsubsection{Hybrid Intelligent Algorithm}

Step 1. Initialize pop_size chromosomes.

Step 2. Calculate the values of (4) for all chromosomes by birandom simulation.

Step 3. Give the rank order of the chromosomes according to the values of (4), and define the rank-based evaluation function of the chromosomes.

Step 4. Compute the value of each chromosome according to the rank-based evaluation function.

Step 5. Select the chromosomes by spinning the roulette wheel.

Step 6. Update the chromosomes by crossover and mutation operations.

Step 7. Repeat the second step to the sixth step for a given number of cycles.
TABLE 1: The distributions of stocks $m_{1}, m_{2}$, and $m_{3}$.

\begin{tabular}{cccc}
\hline Parameters & $m_{1}$ & $m_{2}$ & $m_{3}$ \\
\hline$a_{i}(i=1,2,3)$ & $2 / 3$ & $1 / 2$ & $1 / 3$ \\
$b_{i}(i=1,2,3)$ & $1 / 3$ & $1 / 2$ & $2 / 3$ \\
$\varepsilon_{i 1}(i=1,2,3)$ & $U[0,6 \%]$ & $U[0,8 \%]$ & $U[0,10 \%]$ \\
$\varepsilon_{i 2}(i=1,2,3)$ & $U[-8 \%, 0]$ & $U[-10 \%, 0]$ & $U[-12 \%, 0]$ \\
\hline
\end{tabular}

Step 8. Take the best chromosome as the solution of portfolio selection.

3.5. A Numerical Example. According to $(A 1)$ in Section 3.1, an investor picks three stocks based on three kinds of technical patterns (the probability of rising is larger than, equal to, and smaller than the probability of falling). The stock return follows birandom distribution and the corresponding parameters of each stock are shown in Table 1 . Besides, the investor takes $m=0.03, n=-0.05, \alpha_{1}=0.4, \alpha_{2}=0.8$, and $\beta=0.7$.

Remark 12 . In Table $1, a_{i}, b_{i}, \varepsilon_{i 1}, \varepsilon_{i 2}$ represent the probability of stock rising, the probability of falling, profit distribution, and loss distribution of stock $m_{i}$, respectively.

So model (6) that satisfies the above investment goals is given by

$$
\begin{array}{ll}
\max & \operatorname{Ch}\left\{x_{1} m_{1}+x_{2} m_{2}+x_{3} m_{3} \geq 0.03\right\}(0.4), \\
\text { s.t. } & \operatorname{Ch}\left\{x_{1} m_{1}+x_{2} m_{2}+x_{3} m_{3} \geq-0.05\right\}(0.8) \geq 0.7, \\
& \sum_{i=1}^{3} x_{i}=1, \\
& x_{i} \geq 0, \quad i=1,2,3 .
\end{array}
$$

In order to compare model $(6)$ with the models in $[8,9]$, the models in $[8,9]$ are listed as below:

$$
\begin{array}{ll}
\max & E\left[x_{1} m_{1}+x_{2} m_{2}+x_{3} m_{3}\right], \\
\text { s.t. } & \operatorname{Ch}\left\{x_{1} m_{1}+x_{2} m_{2}+x_{3} m_{3} \geq-0.05\right\}(0.8) \geq 0.7, \\
& \sum_{i=1}^{3} x_{i}=1, \\
& x_{i} \geq 0, \quad i=1,2,3, \\
\max & \operatorname{Ch}\left\{x_{1} m_{1}+x_{2} m_{2}+x_{3} m_{3} \geq 0.03\right\}(0.4), \\
& \sum_{i=1}^{3} x_{i}=1, \\
& x_{i} \geq 0, \quad i=1,2,3 .
\end{array}
$$

The parameters of the hybrid intelligent algorithm are taken as pop_size $=30$, the crossover probability $=0.3$, the mutation probability $=0.5$, the evaluate function based on the order $\alpha=0.05$, and the number of cycles is equal to 1000 . After repeating the hybrid intelligent algorithm 10 times and 
TABLE 2: The results of models (9)-(11).

\begin{tabular}{lccccc}
\hline \multirow{2}{*}{ Model } & \multirow{2}{*}{ Result } & \multicolumn{3}{c}{ Optimal solution } & Objective function \\
& & $x_{1}$ & $x_{2}$ & $x_{3}$ & value \\
\hline Model (10) Result 1 & 0.1916 & 0.6885 & 0.1199 & 0.4157 \\
Model (11) Result 2 & 0.9209 & 0.0668 & 0.0123 & 0.5525 \\
Model (12) Result 3 & 0.0011 & 0.0123 & 0.9866 & 0.6890 \\
\hline
\end{tabular}

taking the average value of the results, the optimal solutions of models (9)-(11) are shown in Table 2.

From result 1 in Table 2, it can be seen that (1) model (10) and the hybrid intelligent algorithm are of great applicability, (2) even if stock $m_{1}$ has the advanced technical pattern, not all investors will buy it using their total asset. Some investors prefer to buy the high-risk and high-interest stock.

From result 2 in Table 2 , it can be seen that the proportion of stock $m_{1}$ is very large. This is because model (11) uses the expected value to measure the profit and stock $m_{1}$ has the largest expected value, so the investor will select stock $m_{1}$ as many as possible, while the constraints are met. However, model (11) is only suitable for risk-neutral investors, so it is restricted in real application.

From result 3 in Table 2, it can be seen that the objective function value of model (12) is larger than it of model (10). This is because model (12) does not consider the risk, so consequently, model (12) has a larger solution space than model (10). Despite achieving the high yield, model (12) is unpractical in application because it ignores the risk.

Through the comparisons of models (10)-(12), it can be concluded that model (10) is more practical than model (11) and model (12), because model (10) not only considers the effect of disaster, but also can apply to all investors through parameters adjustment.

\section{The Effect of Exit Strategy on the Optimal Portfolio Selection}

Exit strategy is a selling strategy including the stop-loss strategy and the stop-profit strategy. If an investor sets the stop-profit point $w \%$ and the stop-loss point $l \%$ for each stock, he will sell the stocks when the stocks' actual profit exceeds $w \%$ or the actual loss exceeds $l \%$. Now we want to ask whether result 1 in Table 2 is still available if the investor set $w=0.4$ and $l=0.5$ for each stock.

we know that in the simulation process of result 1 , the stock return of each stock can be any sample point following corresponding birandom distribution. However, in practice, the cases that actual stock return is over $4 \%$ or below $-5 \%$ will not occur due to the setting of the stop-loss point and the stop-profit point. Thus the calculation of result 1 is inconsistent with the investor's decision-making process. In other words, result 1 cannot reach the investor' objective in theory, which causes the failure of model (10).

In order to solve the above conflict, we should adjust the stock return based on the stop-loss point and the stop-profit point. Considering the assumptions of model (10), we revise the calculation in the following ways.
Step 1. The investor should adjust the stock return according to the stop-profit point and the stop-loss point. Adjustment method is to take the stop-profit point as stock return when stock return exceeds the stop-profit point and to take the stop-loss point as stock return when stock return is less than the stop-loss point. Take stock $m_{1}$ in Table 1 as an example, if an investor set the stop-profit point as $4 \%$ and the stop-loss points as $-5 \%$, the part exceeding $4 \%$ in $\varepsilon_{11}$ is treated as $4 \%$ and the part below $-5 \%$ in $\varepsilon_{12}$ is considered as $-5 \%$.

Step 2. Because of the adjustment of the stock return, we also should modify the steps of birandom simulation. The adjustment method is that if the sample point exceeds the stop-profit point or is less than the stop-loss point, we take the stop-profit point or the stop-loss point as the sample point. The rest of the steps of the hybrid intelligent algorithm remain unchanged. Take stock $m_{1}$ in Table 1 as an example. when samples are selected from $\varepsilon_{11}$, the part which is more than $4 \%$ is looked upon as $4 \%$ and when samples are selected from $\varepsilon_{12}$, the part which is below $-5 \%$ is considered as $-5 \%$.

Through the above adjustment, the calculated value can reach the investor's goal in theory. The revised result of model (10) is

$$
\begin{gathered}
\max C h=0.4908, \\
x_{1}=0.9991, \quad x_{2}=0.0002, \quad x_{3}=0.0007 .
\end{gathered}
$$

It can be seen from result (13) that

(1) because the objective function value in result (13) is larger than it in result 1 , the performance of taking the exit strategy is better than when the exit strategy is not taken, if the stop-loss point is equal to $-5 \%$ and the stop-profit point is equal to $4 \%$;

(2) the exit strategy affects the buying strategy and the investor should adjust the stock return according to the stop-loss point and the stop-profit point, otherwise the portfolio model will become invalid;

(3) the investor using exit strategy become conservative. This is because he is concerned with the accumulated probability of stock price exceeding the stop-profit point not the higher stock return any more.

\section{Conclusion}

In this paper we propose a new method to estimate the stock return and use a birandom variable to denote the final stock return which can reflect the features of technology forms and investors' heterogeneity simultaneously. Besides, we build a birandom safety-first model and design a hybrid intelligent algorithm to help investors make a decision. Moreover, we innovatively study the effect of exit strategy on the given birandom safety-first model. The results indicate that the performance of taking the exit strategy is better than when the exit strategy is not taken, if the stop-loss point and the stop-profit point are appropriately set. 


\section{Appendix}

\section{Proof of Remark 8}

Let $A$ represent the solution space which satisfies

$$
\mathrm{Ch}\left\{x_{1} m_{1}+x_{2} m_{2}+\cdots+x_{n} m_{n} \geq n\right\}\left(\alpha_{2}\right) \geq \beta_{1} .
$$

Let $B$ represent the solution space which satisfies

$$
\operatorname{Ch}\left\{x_{1} m_{1}+x_{2} m_{2}+\cdots+x_{n} m_{n} \geq n\right\}\left(\alpha_{2}\right) \geq \beta_{2},
$$

and $\beta_{1}>\beta_{2}$.

For any $x=\left(x_{1}, x_{2}, \ldots, x_{n}\right) \in A$. If $\omega=\left(\omega_{1}, \omega_{2}, \ldots, \omega_{n}\right) \in$ $\Omega$ satisfies

$$
P_{r}\left\{x_{1} m_{1}\left(\omega_{1}\right)+x_{2} m_{2}\left(\omega_{2}\right)+\cdots+x_{n} m_{n}\left(\omega_{n}\right) \geq n\right\} \geq \beta_{1}
$$

then we can obtain that $\omega=\left(\omega_{1}, \omega_{2}, \ldots, \omega_{n}\right) \in \Omega$ also satisfies

$$
P_{r}\left\{x_{1} m_{1}\left(\omega_{1}\right)+x_{2} m_{2}\left(\omega_{1} 2\right)+\cdots+x_{n} m_{n}\left(\omega_{n}\right) \geq n\right\} \geq \beta_{2} .
$$

So if $x=\left(x_{1}, x_{2}, \ldots, x_{n}\right) \in A$ satisfies

$$
\begin{array}{r}
P_{r}\left\{\omega \in \Omega \mid P_{r}\left\{x_{1} m_{1}\left(\omega_{1}\right)+x_{2} m_{2}\left(\omega_{1} 2\right)+\cdots\right.\right. \\
\left.\left.+x_{n} m_{n}\left(\omega_{n}\right) \geq n\right\} \geq \beta_{1}\right\} \geq \alpha,
\end{array}
$$

then we can obtain $x=\left(x_{1}, x_{2}, \ldots, x_{n}\right)$ satisfies

$$
\begin{array}{r}
P_{r}\left\{\omega \in \Omega \mid P_{r}\left\{x_{1} m_{1}\left(\omega_{1}\right)+x_{2} m_{2}\left(\omega_{1} 2\right)+\cdots\right.\right. \\
\left.\left.+x_{n} m_{n}\left(\omega_{n}\right) \geq n\right\} \geq \beta_{2}\right\} \geq \alpha .
\end{array}
$$

Since (A.6) is equivalent to (A.2), so $x=\left(x_{1}, x_{2}, \ldots, x_{n}\right) \in$ $B$. Hence, $A \subset B$. That is to say, the value of $\beta$ is in inverse ratio with the range of solution space.

Since when the range of solution space is enlarged, an investor has a larger decision range, which means he/she can try some investments that others dare not try, so the risk tolerance ability is increased vice versa.

So the range of solution space is in direct ratio to risk tolerance ability; therefore, the value of $\beta$ is in inverse ratio with risk tolerance ability.

\section{References}

[1] M. Douglas, Trading in the Zone, Prentice Hall, New York, NY, USA, 1st edition, 2000.

[2] H. Markowitz, "Portfolio selection," Journal of Finance, vol. 7, no. 1, pp. 77-91, 1952.

[3] D. Da-Yong and J. Wei-Dong, "A subjective model of the distribution of returns and empirical analysis," Chinese Journal of Management Science, vol. 15, pp. 112-120, 2007.

[4] L. Mei-Yan, X. Hong-Gang, and Z. Feng-Qun, "Statisticsal analysis of return rates of shanghai stock market price integrated index," Operations Research and Management Science, vol. 14, pp. 115-119, 2005.
[5] F. Jian-Qiang and W. Fu-Xin, "A research on return distribution function of chinese stock-market," Chinese Journal of Management Science, vol. 11, pp. 82-90, 2007.

[6] J. Li, J. Xu, and M. Gen, "A class of multiobjective linear programming model with fuzzy random coefficients," Mathematical and Computer Modelling, vol. 44, no. 11-12, pp. 1097-1113, 2006.

[7] X. Huang, "A new perspective for optimal portfolio selection with random fuzzy returns," Information Sciences, vol. 177, no. 23, pp. 5404-5414, 2007.

[8] L. Mei-Yan, "Chance-constrained portfolio selection with birandom returns," Modern Applied Science, vol. 3, no. 4, pp. 161$165,2009$.

[9] L. Mei-Yan, "One type of optimal portfolio selection in birandom environments," Modern Applied Science, vol. 3, no. 6, pp. 121-126, 2009.

[10] A. D. Roy, "Safety-first and the holding of assets," Econometrica, vol. 20, no. 3, pp. 431-449, 1952.

[11] D. H. Pyle and S. J. Turnovsky, "Safety-first and expected utility maximization in mean-standard deviation portfolio analysis," The Review of Economics and Statistics, vol. 52, no. 1, pp. 75-81, 1970.

[12] Z. Yong-Fen, "Based on the safety first chance constrained dynamic portfolio research problems," Modern Economic Information, vol. 18, pp. 32-33, 2009.

[13] P. Jin and L. Bao-Ding, "Birandom variables and birandom programming," Computers and Industrial Engineering, vol. 53, no. 3, pp. 433-453, 2007. 


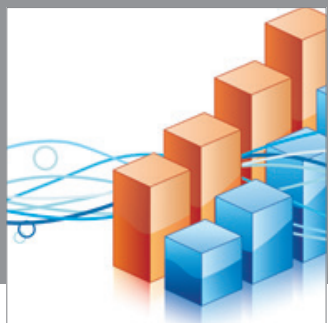

Advances in

Operations Research

mansans

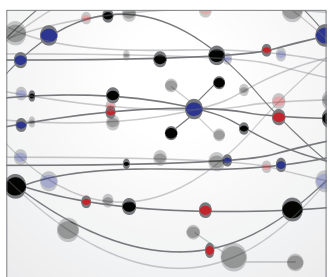

The Scientific World Journal
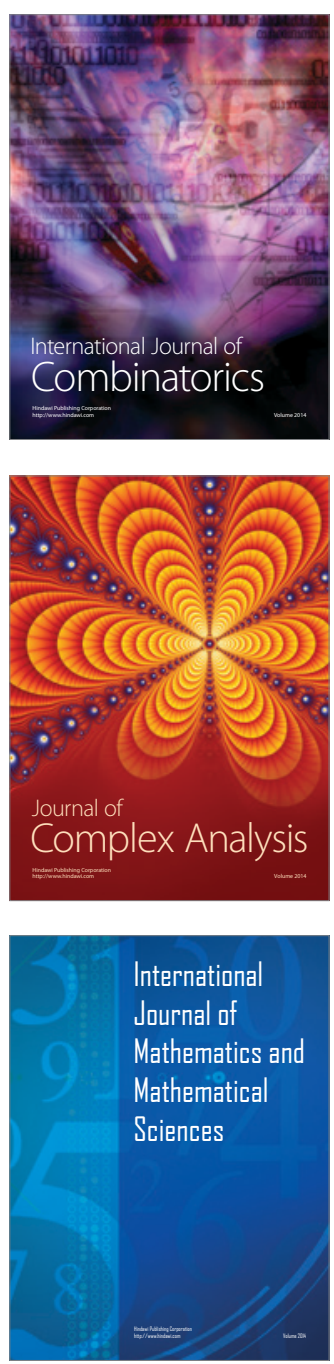
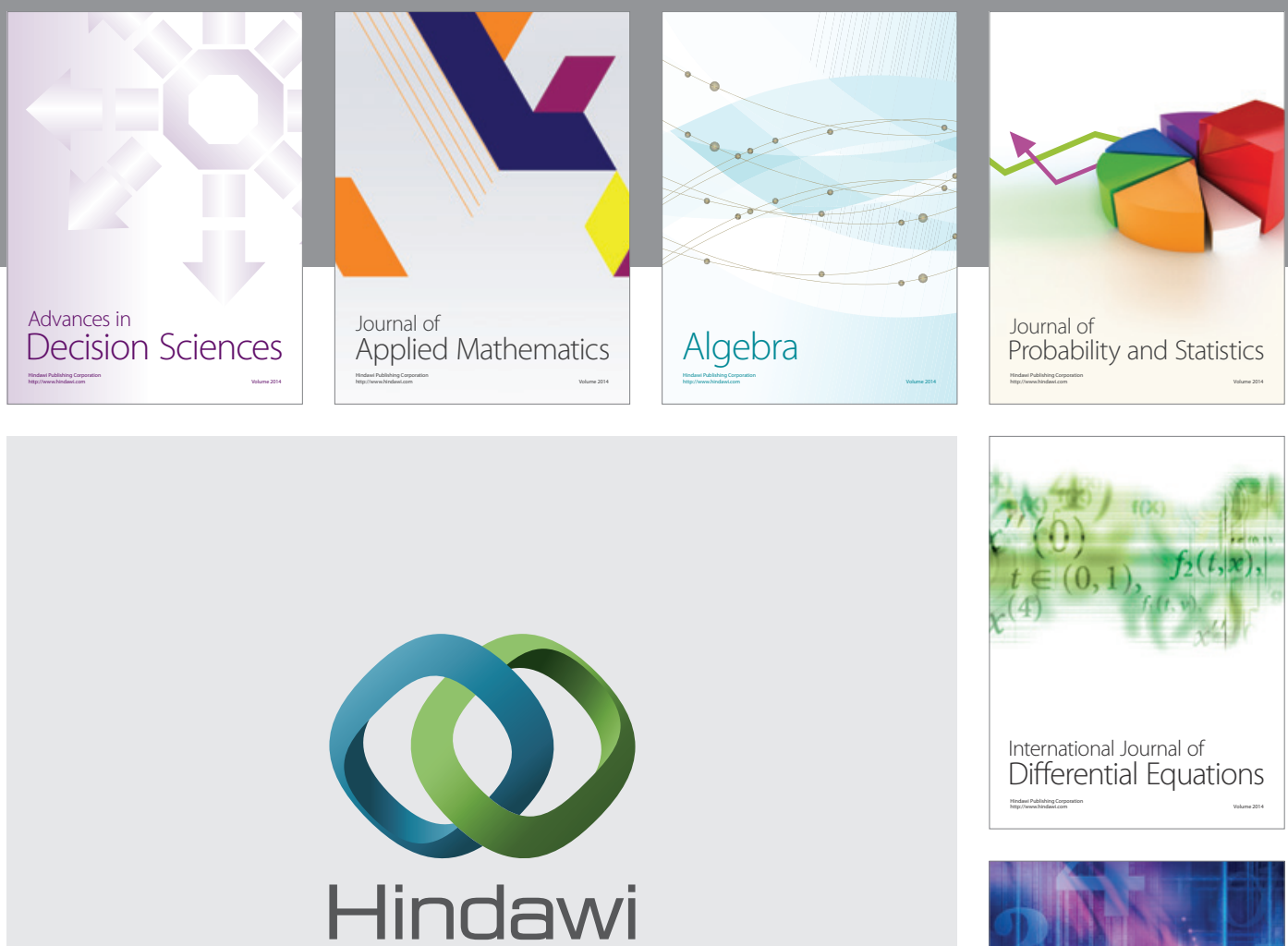

Submit your manuscripts at http://www.hindawi.com
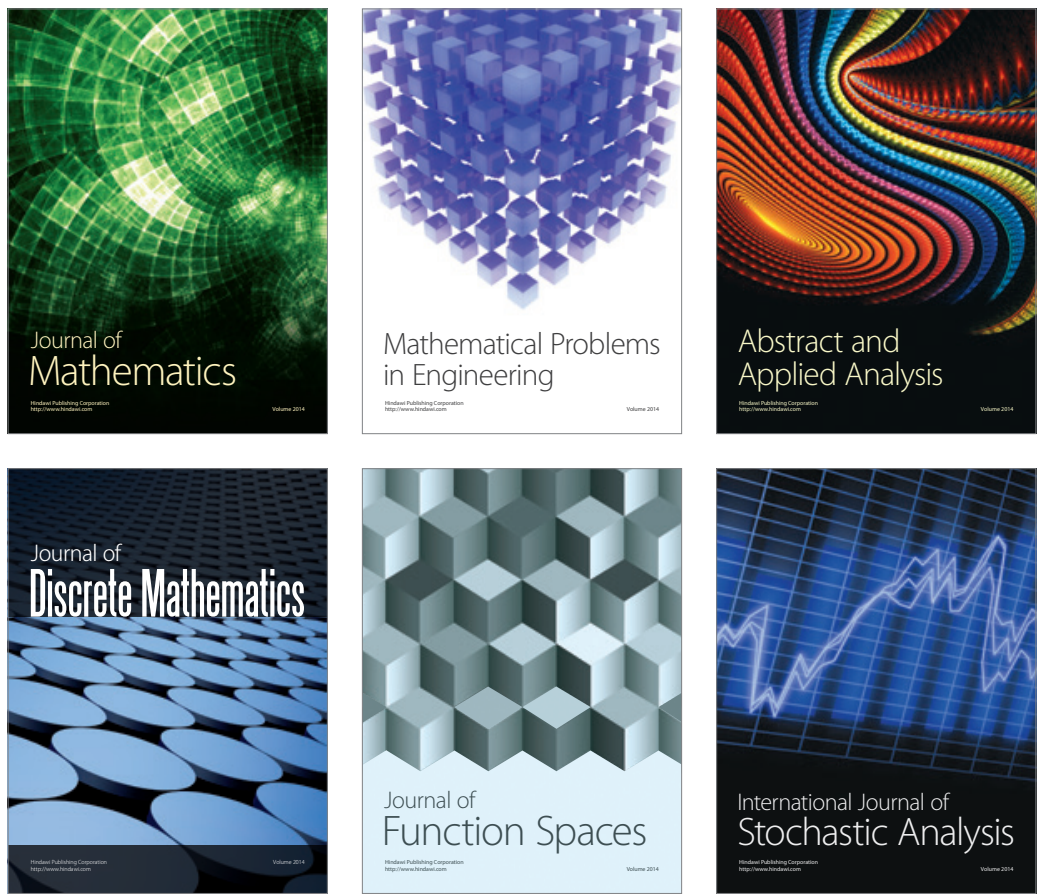

Journal of

Function Spaces

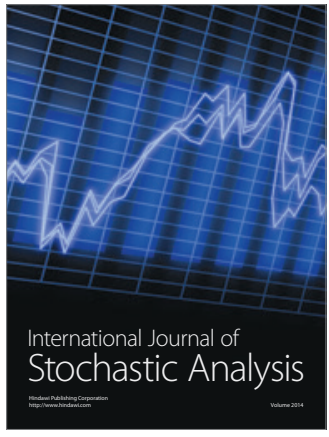

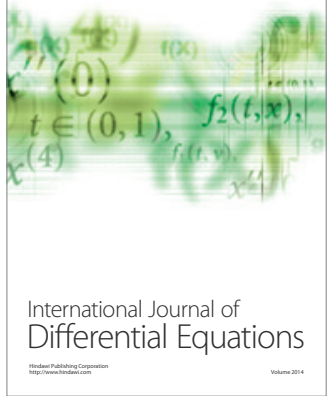
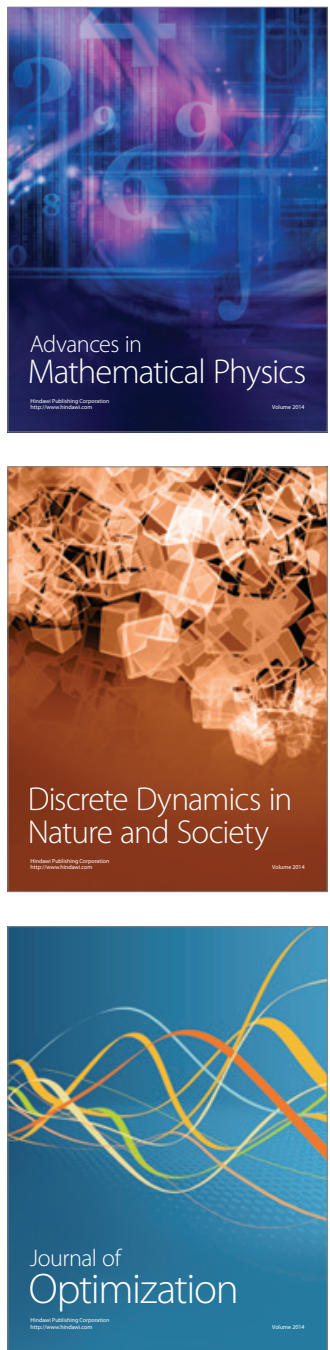